\title{
Elementary forms of linguistic organisation
}

\author{
Wolfgang Klein
}

What song the Sirens sang, or what name

Achilles assumed when he hid himself among women, although puzzling questions, are not beyond all conjecture.

Sir Thomas Browne

\section{Introduction}

Studying the past is dull, dusty, and difficult and does not seem to provide a selective advantage. So why do we do it? The first and most obvious reason is simple curiosity. With the thrill of children exploring grandmother's attic, we dig among the material and immaterial remnants of past worlds. This is a respectable motive. After all, curiosity is at the origin of all research, of all systematic attempts to understand the world around us.

The second reason is the old idea that the truth can be found in the past. It is perhaps not accidental that the study of language began with the quest for the origin of words - etymology. The idea was that things had "right" names. If you wanted find out the truth about something you had to uncover the real meaning of its name. Etymologies have since become less important. Men named "George" are no longer suspected of being peasants in disguise. Nevertheless, etymologies often seem to contain at least a grain of truth. According to Grimms' dictionary - one of the greatest scientific achievements of the Berlin Academy and of lexicographical research ever the German word Ehe ('marriage') is derived from the Germanic word aivs meaning 'eternity'. The English word ever has the same etymon. And aren't marriages supposed to last forever?

The third reason is that we tend to believe that studying the past helps us to understand the present. Examining the present can tell us how things are, but not why they are as they are. This notion is not new. But it was only during the nineteenth century that it became a key concept of numerous scientific disciplines. In biology, the transition from static Linnean classification to evolutionary dynamism is a case in point. It was no less common in the scientific investigation of language. To Hermann Paul, whose 1880 


\section{Wolfgang Klein}

Principien der Sprachgeschichte marked the culmination of nineteenth-century linguistic thought, it was evident that there can be only one scientific approach to language: diachronic analysis. Studying the present may well uncover facts, but it cannot explain them. It is thus not truly scientific:

Es ist eingewendet, daß es noch eine andere wissenschaftliche Betrachtung der Sprache gäbe, als die geschichtliche. Ich muss das in Abrede stellen. Was man für eine nichtgeschichtliche und doch wissenschaftliche Betrachtung der Sprache erklärt, ist im Grunde nichts als eine unvollkommen geschichtliche, unvollkommen teils durch Schuld des Betrachters, teils durch Schuld des Beobachtungsmaterials. Sobald man über das blosse Konstatieren von Einzelheiten hinausgeht, sobald man versucht, den Zusammenhang zu erfassen, die Erscheinungen zu begreifen, so betritt man auch den geschichtlichen Boden, wenn auch vielleicht ohne sich klar darüber zu sein.

[It has been objected that there might be a scientific approach to language other than the historical one. I must refute this claim. What has been declared to be an ahistorical but nevertheless scientific approach to language is in actual fact nothing more than an imperfect historical approach - imperfect due partially to insufficiencies on the observer's part and partially to insufficiencies in the material under observation. As soon as we go beyond mere details, as soon as we attempt to grasp the relations among phenomena and to understand them, we enter the realm of history, albeit perhaps without being aware of it]

(Paul 1886: 19-20, my translation).

A century later we are less convinced that a truly scientific analysis must be historical. There are explanatory factors beyond evolution, in biology as well as in linguistics. Still, the idea that to understand things we must examine how they came to be is deeply rooted in our thought. It is this idea that makes us feel that the study of how mankind came to language - or language to mankind - is more than a matter of mere curiosity.

But there are two fundamental problems with this idea. First, the word "language" is used in many ways. It is consequently not clear what we mean when we talk about the origin of language. Second, there is something paradoxical about the idea that we should study the past in order to understand the present. After all, the past is past, and we have no access to it. What we have access to are its remains: bones, teeth, and petrified foot prints. And of course we have ourselves, products of past development. When we study the past to understand the present, what we are really doing is studying selected aspects of the present in the hope that this might help us to understand the past. If we want to know how language came about, there are only two paths to take. We can reconstruct it from what we have here and now as the 
result of past development. Or we can look at cases in which similar processes are occurring here and now. But are there such cases? The answer depends on what we mean by language and language origin.

\section{Three notions of language and language origin}

Ever since Ferdinand de Saussure at the beginning of the last century, linguists distinguish among at least three types of linguistic facts.

First, there are facts that are characteristic of the ability to create, learn, and use particular languages. Saussure called this ability the faculte de langage or simply langage. I will use the term "human language faculty". It is the faculty with which normal human beings are born; it belongs to our genetic endowment. This fact is beyond doubt, as is the fact that it must somehow be part of our nervous system and our body. But there are also a number of unresolved questions:

1. Is the language faculty specific to our species or is it also found in other animals like higher primates? And if so, why don't they typically use it?

2. Is it domain-specific? That is, is it just one of the many aspects of human memory and cognition in general, or is it a separate module in our brain?

3. How does it develop over one's life span? Is it fully developed at birth or does it develop over time? Does it deteriorate with age? And if so, when and at what rate?

These are not easy questions. But they are sufficiently well defined to be investigated scientifically.

Second, though the language faculty is part of our genetic endowment, we are not born with a language. The capacity as such is not enough. Children (and adults under appropriate circumstances) must learn a particular language like Russian, Urdu, or Kpelle - a langue, as Saussure said. A langue is a system of expressions with specific properties. Linguists disagree to some extent on what these properties are and how such a system should be analysed. But they concur on two points. First, a linguistic expression is a particular combination of a form (usually a sequence of sounds) and a meaning. Second, there are elementary expressions (words), and there are complex expressions formed by certain morphological and syntactical operations (phrases, sentences, and 


\section{Wolfgang Klein}

texts). In short, every language has a lexicon (an inventory of elementary expressions) and a grammar (rules according to which words can be modified and put together to form extended expressions). There are also a number of questions concerning the properties of these systems:

1. What does the - the most common word of the planet's most-spoken language - mean?

2. What is the function of inflectional morphology?

3. Why is possible to say the only book, but not an only book or three only books?

4. How does the meaning of the complex expression the only book result from the meaning of its parts?

There are several thousands of these systems across the world. They differ considerably. But diversity does not mean that there are no shared characteristics. In fact, there may be properties - linguistic universals - that are found in all pairings of sounds and meanings. It makes sense to assume that these universals reflect properties of our innate language faculty. In other words, the properties of the human language faculty and the properties of specific languages - linguistic systems - must be related to each other.

The third set of linguistic facts comprises what Saussure called parole, the actual communication between human beings in a given situation: gossiping, cursing, praying, holding lectures, describing a living room, and arguing about the origin of the language faculty. With some exceptions (like keeping a diary), linguistic communication involves more than one participant. There are a number of typical questions about parole:

1. Beyond the sound-meaning pair in question, which other components of the human mind - memory, reasoning, and so forth - play a role in communication?

2. What adaptive value does linguistic communication have for the individual and for his or her social group?

3. How does communication by sound-meaning coupling interact with other forms of human communication like facial expressions and gestures?

4. How does human communication differ from communication among bees, dolphins, or lobsters? 
These are also difficult questions. But they too can be investigated empirically, and much has been learned about them. The third type of linguistic phenomenon is the most complex. It has physical components like the acoustics of the room or the properties of the paper on which something is written. It has biological components like the participants' voice properties. It has social components like the personal relations among speakers. And it has cognitive components like the spatial knowledge needed to give directions or the planning capacity needed to construct a coherent text.

What do we mean when we talk about language origin? Do we mean the origin of the human language faculty, the origin of the first linguistic system, or the origin of communication with the aid of such a system? It is important to remember that these are quite different notions of origin.

The language faculty is part of our biological equipment. At some point in history, it was formed by genetic changes involving several parts of the human body. Some of the changes affected our nervous system where the central part of the language faculty is stored. Others affected peripheral organs like the larynx. We do not know when these changes occurred. And we do not know how and when they were synchronised into the capacity that is now standard human equipment. Regarding the central part of the language faculty, we do not know whether there was a single change or a whole series of them. Nor do we know whether the change or changes only produced the human language faculty or whether they are also responsible for other brain capacities.

All the human language faculty needed was a language. But no such linguistic system was available, so the language faculty had to create one. This is the second meaning of language origin. The creation of the first sound-meaning coupling is very different from the formation of the human language faculty. We do not know how long the transition period was. There may have been hundreds of thousands of years between the origin of the language faculty and the origin of the first language. This raises a number of empirical and theoretical issues.

First, was there one first language or did the language faculty independently create several first systems? The longer the time lag between the origin of the language faculty and its manifestation in a language, the likelier it was that several such systems were created independently. But we simply do not know. Structural equivalencies across languages are not evidence for a monogenetic origin, since they may simply reflect properties of the language faculty itself. 


\section{Wolfgang Klein}

Second, what is the selective advantage of having a language faculty without a language? Such a major component of the brain consumes a lot of energy. And as long as it is not used - and it cannot be used if there is no language - it is anything but an advantage. A language faculty without a language would be a parasite unless it also served other functions. Alfred Wallace was the first to raise this issue for cognition in general. He proposed that the early growth of the human brain represented a moral rather than intellectual advantage. Charles Darwin did not share Wallace's rosy view, but he could not supply a satisfactory answer. In fact, he told Wallace that he was about to kill "your own and my child" (quoted in Desmond and Moore 1991: 642). And there is still no answer, at least with regard to language.

Third, what did the first system (or systems) look like? We do not know. It seems unlikely that it was as complex as the first well-documented languages like Sanskrit. Maybe it was more like a pidgin. In order to qualify as a human language, it must have had a lexicon and a grammar. Is there is a "basic" lexicon or a "basic" grammar? How are they related to each other? Is grammar just a projection of the lexicon (as some linguists propose) or is it a completely different component? And is there any way to answer these questions?

Fourth, how did it come into more than one head? The birth of the language faculty was a biological process, and it spread among individuals via a biological process. But the fact that the sound sequence /ho:mo:/ is associated with a particular meaning - and the fact that /ile ho:mo: pater me:us est/ is composed as it is and means what it means - is not biologically transmitted. We know it because we have learned it from others via experiential transmission: by ears and eyes rather than by genes. But this mode of transmitting linguistic knowledge cannot have applied to the first linguistic system. It was the joint creation of a social group that somehow agreed that a particular sound sequence is systematically coupled with a particular meaning and must be combined in a particular way with other sound sequences to form more complex expressions. How was this possible? Again, we do not know. But we do know that in contrast to the origin of the linguistic faculty, the origin of linguistic systems has a fundamentally social dimension.

As soon as there was a first language it became possible to convert thoughts, feelings, and wishes into sound waves, to transmit them to others, and, in turn, to influence their thoughts, feelings, wishes, and behaviour. It is the use of a linguistic system that orients human beings in their environment differently from a monad in a world defined by the laws of pre- 
established harmony and from an ant in a world ruled by the rigid interactional principles of an ant colony. The verbal transmission of theoretical, practical, and situation-bound knowledge from one generation to the next sets the stage for the particular type of behaviour we consider human. It is the use of language that makes possible all higher forms of cognition as well as the characteristically human kind of interaction between members of the species. The crucial point here is that neither the mere existence of the biologically given human language faculty nor the mere existence of a first linguistic system suffices to achieve this - for the simple reason that linguistic communication involves more. It requires a complex interplay of various cognitive and social capacities. These include storing certain types of knowledge, appropriately selecting pieces of this knowledge, integrating expressions into an ongoing flow of information, and adapting to a social environment. This is the third meaning of language origin: the origin of (linguistic) communication. It presupposes the two other origins, but goes far beyond them (just as it goes far beyond other kinds of communication that do not require a linguistic system). It is only this type of language that has adaptive value.

Linguists are primarily interested in the second notion of language: in the structure and functioning of linguistic systems. In fact, linguists cannot contribute much to answering questions about the origin of the language faculty, unless it is by pointing out which properties biologists and brain researchers ought to consider. The knowledge of a few signs does not require something as complex as the human language faculty. Dogs and cats have them or can learn them. So what is it that distinguishes human languages from other systems of communication?

\section{Lexical repertoire and rules of composition}

Two notions seem uncontroversial among linguists. First, there must be a set of elementary expressions (lexemes). Second, there must be rules of composition that prescribe how complex expressions are formed from simpler ones. In other words, there must be lexicon and a grammar. This applies to all manifestations of the human language faculty, from elementary learner varieties (like the language of beginning second language learners or of pidgin speakers) to fully fledged languages like Latin or English. 


\section{Wolfgang Klein}

\subsection{The lexicon}

A lexeme (word) is a cluster of at least three types of features: semantic features that indicate an expression's lexical meaning (or "lexical content"); phonological features that describe an expression's phonological shape; and categorical features that characterise an expression's behaviour with respect to rules of composition. Let us consider the English lexeme horse. The visual shape on the paper is not the word, but rather it is one way of representing the word. The word itself is a cluster of features. They include the phonological information $/ \mathrm{h}: \mathrm{s} /$, the semantic information "equine quadruped", and the categorical information "is a noun". Horse is a simple example, and things can get more complicated. In particular, other properties - graphematical features - may be linked to a lexeme. But they are not crucial (written language is a relatively late invention, and many, if not most, languages still lack it). There are many lexemes whose semantic properties are far more abstract than horse. We might consider the English morpheme the, the most frequent lexical item on earth. No linguist has managed to give a precise and clear characterisation of what it means. Bertrand Russell, for example, begged his readers not to reject his theory for its "apparently excessive complication" until they themselves had "attempted a theory ... on the subject of denotation" (Russell 1905: 493). In addition, linguists largely agree that in some specific cases semantic features can be entirely absent, as in the case of there in there is a slug in the salad. Similarly, phonological information can be absent like in the case of socalled "empty elements". What seems indispensable are categorical features: each lexical item must contain information about how it can be integrated into larger constructions.

The first task the human language faculty must be able to perform is to create a lexicon. This means that it must be able to sort out these three types of features (semantic, phonologic, and categorical), to cluster them in some fashion, and to store them somewhere in the brain. There are two ways to achieve this: either by copying an existing repertoire or by creating new clusters. Today, with so many languages available, the first way predominates. It constitutes the lexical part of language acquisition and is an extremely complicated process, many aspects of which are still a complete mystery (see Clark 1993). Children or adult learners are not confronted with words, but with more or less continuous sound streams that are initially baffling. They must break such sound streams into smaller segments and associate these segments with semantic features and - what is more difficult - 
categorical features. This process, though little understood, is highly efficient. Children learn thousands of words within a few years. And although the capacity to learn new lexical items seems to deteriorate with age, it is hardly ever lost entirely.

When our ancestors had the language faculty but no language, they had to invent lexemes. No one knows how they did it. But it was and still is a fertile field for speculation (see the survey in Kainz 1967: 267-334). Did the first lexemes express emotions like pain or fear? Did they imitate natural sounds? It is difficult to imagine how one might arrive at even a simple lexeme like tree. We simply have no historical evidence. But we can examine instances where the human language faculty still creates new lexical items. These cases are admittedly infrequent. Children sometimes create lexical items that are not based on existing words. Psycholinguists invent nonce words for experimental purposes. Pharmaceutical companies design names for new products. These names are often based on Latin or Greek roots, but are just as frequently new coinages. In German, the lexeme hungrig ('hungry') has an antonym that describes the state of having had enough food. The lexeme durstig ('thirsty') lacks such a counterpart. German has a lexical gap. We could of course fill it by a new lexeme, say schwock. (English even lacks an antonym for hungry.) What is characteristic of all observable cases of lexeme creation is that they begin with semantic features and associate them with phonological features. Was this also true for our earliest ancestors? It should be true unless we assume that the human language faculty itself has changed. But if the human language faculty has changed, then it is hopeless to say something about its origin.

So if we want to understand the past, we must study the present. The creation of new lexical items is perhaps not the best example for this strategy. Children perfectly imitate the language around them. And when adults create a new item they do so in the context of a language or languages they already know. Nevertheless, I believe this is the only realistic approach. So far I have not addressed the way categorical features are added to a lexeme. It is a complete mystery. Most linguists believe that semantic and phonologic features can be absent from a lexeme. Categorical features, however, must be present. These features reflect the combinatorial side of a linguistic system: its grammar. 


\subsection{Rules of composition}

Grammatical rules are traditionally divided into morphological and syntactical rules depending on whether they operate within or go beyond individual words. There are a number of borderline cases (just as there are borderline cases between lexicon and grammar). The most salient example of morphological rules is inflection. The Western linguistic tradition has frequently equated inflection with inflectional morphology. This is because the languages that stood at the beginning of this research tradition - Greek and Latin - have rich inflectional systems. The first grammars of comparatively modern languages like English and German readily adopted this "morphology bias". The fact that they had less elaborate inflectional systems than classical languages was generally seen as a symptom of decay. This view is today obsolete. Inflectional morphology is a common but by no means indispensable aspect of languages. In other words, there is grammar beyond inflection. But as strange as the notion of grammatical decay may seem, it is a fact that to the extent to which we have historical records languages tend to reduce or to eliminate inflectional morphology rather than to expand it. There are some exceptions, such as the formation of future marking in Romance languages (aimerai from amare habeo). Although frequently referred to, these cases are rare and do not affect the overall picture. English, Dutch, and even German show very reduced inflectional systems when compared with their common Westgermanic origin, let alone when compared with older stages of Indo-European. As August Schleicher pointed out many years ago, English had is the modern equivalent to Germanic (more precisely Gothic) habaidedema. Chinese, the paradigmatic example of a language without inflection, is assumed by many scholars to have had suffixes at one time. But they are gone and have only left behind traces in the form of lexical tones. So, despite some exceptions, languages seem to develop away from morphology. This fact led Otto Jesperson to assume that in the beginning languages were much more complex than they are today: "we must imagine primitive language as consisting (chiefly at least) of very long words, full of difficult sounds, and sung rather than spoken" (Jesperson 1921: 421). His theory is based mainly on the development of languages for which we have written records. He sums up his argument with a general "law of development": "The evolution of language shows a progressive tendency from inseparable irregular conglomerations to freely and regularly combinable short elements" (Jesperson 1921: 429). 
This notion is counterintuitive. It is hard to imagine that our ancestors who designed the first linguistic system immediately created something as morphologically complex as Sanskrit. It seems more likely that the first systems had no complex morphology and only very elementary combinatorial rules. Again, the only way to find out is to examine situations in which the human language faculty does not copy an existing system but somehow creates elementary combinatorial rules.

The distinction between morphological and syntactic rules is generally accepted in modern linguistics. There is also agreement that the latter are in a way more fundamental. After all, there are linguistic systems that keep lexemes intact, but there are no languages that do not allow the formation of expressions consisting of several separate lexemes. The next point is more controversial. I would like to make a rigid distinction between two types of rules of composition. There are rules that operate on lexical contents, and there are rules that serve to integrate an expression into a given context. I will call the former LC rules and the latter CI rules. LC rules serve to form complex lexical contents from simple ones. By doing so, they affect an item's semantic, categorical, and phonological features. For example:

1. The accusative singular of German nouns of paradigm class five is formed by attaching $-\mathrm{n}$. This is a morphological rule based on categorical features.

2. A lexeme of the type "determiner" and a lexeme of the type "noun" form an expression of the type "noun phrase". This is a syntactic rule based on categorical information.

3. The constituent that expresses the agent comes first. This is a syntactic rule based on semantic features.

Compositional rules can also - perhaps only - use the semantic information provided by lexemes rather than by categorical features. There are also compositional rules (such as French liaison) that only affect phonological information. All that matters is that they are stated in terms of the information provided by the lexemes involved.

Let us turn now to the rules that integrate the utterance into the ongoing flow of contextual information. Such information may stem from preceding utterances, from the speech situation, or from the speaker's or listener's general world knowledge. Typical CI rules might be: 


\section{Wolfgang Klein}

1. Focus constituents come last.

2. Lexemes that preserve information from the preceding sentence come first.

3. Lexemes that preserve information from the preceding sentence are de-accented. They also include rules regarding the communicative function, in particular a sentence's "illocutionary force" when it is uttered in a certain communicative context:

4. A question is marked by a final rise

5. An assertion is marked by having the finite component of the verb in second position

6. An imperative is marked by a bare stem in initial position.

Rules like the six above are not based on lexical information. After all, nothing in the meaning of the lexeme come says that it should be used as a question, an assertion, or an imperative, just as nothing in the lexical information of this lexeme tells us whether, in a given utterance, this information is new or retained from a preceding utterance.

The distinction between LC rules and CI rules is not absolute. It does not preclude that a given language will cluster bits and pieces of both types together into a single complex rule. In fact, the apparent opacity of "fully fledged" languages is often due to such clustering, whereas the distinction is clearer in more elementary manifestations of the human language capacity. In the latter, we seem to have very simple rules such as "agent first" (an LC rule) or "focus last" (a CI rule). The problem is that under specific communicative circumstances the two types of rules come into conflict. Hence, when applied simultaneously, they do not allow the formation of a complex expression. Such cases call for additional devices. Indeed, this may be the driving force behind the development of linguistic systems that go beyond mankind's first languages.

There is another reason why the distinction between LC rules and CI rules is important in the current context. It may be that these two types of compositional rules belong to different components of the human language faculty. In fact, they may have developed at different times. There was consequently not just a single period during which this faculty developed. We intuitively assume that the capacity to integrate meaningful stretches of sound into the ongoing flow of discourse precedes the capacity to build complex constructions based on the categorical information of lexemes. But we do not know for sure, and there is no easy way to find out. Again, all we can do is examine how this faculty currently functions when it goes about developing elementary linguistic systems. 


\section{The Basic Variety}

If the human language faculty has undergone substantial changes since the days when it created the first linguistic systems, then there is little hope of finding out what it was like in those days or what these first systems looked like. If it has not undergone substantial changes, then we should examine how it currently operates when faced with a similar task. Such situations seem rare; the case which comes closest to them is language acquisition. We all are born with the language faculty, but not with a particular language. Hence, while mankind has many languages, each newborn has to go through a "latent language faculty" period in which he or she has the faculty but not the linguistic system. This case is substantially different, since today our innate faculty does not have to invent lexemes and rules of composition, but rather copy them from an existing language. This is also true for second language acquisition (SLA). But isn't it at least possible that the process of language acquisition displays aspects that are independent of the particular language being learned? No one seems to have made this claim for first language acquisition. As a rule, children can copy even the most irrelevant details of the language they are learning. True, there might be creative aspects to the acquisition process, but they are not obvious. The language-copying component of children's language faculty seems to outweigh - or render superfluous - the language-making component. This may be different for adult second-language learners, since they are less able or less willing to copy what they hear. The linguistic systems adult learners develop - their learner varieties lag considerably behind the target language they are trying to replicate. This may be because they have already mastered a language - their source language - or because of changes in their learning capacity. Teachers, linguists, and laymen tend to view these learner varieties as imperfect replications of the target. But to the extent that such systems also exhibit properties that are independent of the source and target languages, we must assume that they reflect creative processes of the underlying human language faculty. Is there such an overarching linguistic system whose structural properties are independent of a particular language?

In a large cross-linguistic and longitudinal research project, we examined how 40 adult learners picked up the language of their social environment via everyday communication (Perdue 1993 contains a detailed description of this project, which involved about fifteen researchers across Europe). Their production was regularly recorded and analysed over about 30 months. This production and the way it evolved varied in many respects. But it also manifested a number 


\section{Wolfgang Klein}

of striking similarities. One of the core findings was the existence of a special language form that we called the Basic Variety (Klein and Perdue 1997). It was developed and used by all learners, independent of source and target language. About one third of the learners fossilised at this level. This means that, minor variations aside, they only extended their lexical repertoire and learned to make more fluent use of the Basic Variety. But they did not complicate their utterances in other ways, particularly with respect to morphology or syntax.

We believe the Basic Variety not only plays a particular role in the SLA process, but also that it represents a particularly natural and transparent interplay between function and form in human language. In a way, fully fledged natural languages are only elaborations of the Basic Variety. They add specific devices like inflectional morphology or focus constructions. They also add numerous decorative elements: pleasant to the ear, hard to learn, and faithfully transmitted from one generation to the next. But fully fledged languages essentially build on the same organisational principles.

\subsection{The Basic Variety's lexicon}

There is no inflection in the Basic Variety, hence no morphological marking for case, number, gender, tense, aspect, or agreement. Typically, a single form corresponds to the stem, the infinitive, or the nominative in the target language. But it can also be a form that would be an inflected form in the target language. Sometimes a word appears in more than one form. Such variation does not seem to have a function; the learners simply try out phonological variants.

The Basic Variety's lexicon varies in size and origin. Normally, it expands steadily during the acquisition process, though this increase varies considerably among learners. The main source is normally the target language. There are also numerous borrowings from the source language. Interestingly, the composition of the lexicon is remarkably constant among all learners. It essentially consists of a repertoire of noun-like and verb-like words as well as a few adjectives and adverbs. The pronoun system is extremely elementary. It includes minimal means to refer to speaker, listener, and a third person (functioning deictically and anaphorically). There are a few quantifiers, a word for negation, and a few prepositions with over-generalised lexical meanings. There are no subordinating conjunctions. In other words, the repertoire consists mainly of openclass items and a few closed-class items with lexical meaning. There are some determiners (in particular demonstratives), but hardly ever a determiner system. And there are no expletive elements like the English existential there. 
What can these findings teach us about the language-making side of the human language capacity? Not much. Most items are replications of something that already exists. What is telling, though, is the absence of some items that are typically found in fully fledged languages, notably semantically empty elements and closed-class items. Both facts are related to categorical properties, that is, to properties that link lexical items with rules of composition.

\subsection{The Basic Variety's rules of composition}

How do speakers of the Basic Variety integrate their repertoire of lexemes into full utterances? The first and most salient point is the already noted absence of verb or noun inflection. There are no morphological rules (though there are some noun-noun compounds). But speakers of the Basic Variety are able to construct more complex utterances. The structure of these utterances is determined by the interaction of three types of constraints. First, there are absolute, "phrasal" constraints on the form and relative order of constituents (these are LC rules based on lexemes' categorical features). Second, there are "semantic" constraints relating to the case-role properties of arguments (these are LC rules based on lexemes' semantic features). Third, there are "pragmatic" constraints relating to the organisation of information in connected text (these are $\mathrm{CI}$ rules for the introduction and maintenance of reference as well as for topic-focus structure). The phrasal constraints observed in the Basic Variety allow three basic phrasal patterns with some subvariants (the subscripts correspond to differences in their possible internal structures):

$$
\begin{array}{lc}
\text { PHla. } & \multicolumn{1}{c}{\mathrm{NP}_{1}-\mathrm{V}} \\
\text { PH1b. } & \mathrm{NP}_{1}-\mathrm{V}-\mathrm{NP}_{2} \\
\text { PHlc. } & \mathrm{NP}_{1}-\mathrm{V}-\mathrm{NP}_{2}-\mathrm{NP}_{2}
\end{array}
$$

$$
\text { PH2. } \quad \mathrm{NP}_{1}-\text { COPULA - } \mathrm{NP}_{2} \text { ADJECTIVE }
$$

PREPOSITIONAL PHRASE

PH3.

$$
\text { V }
$$




\section{Wolfgang Klein}

All patterns may be preceded or followed by an adverbial phrase (normally one of time or place) or by the conjunction and.

The phrasal constraints impose narrow restrictions on possible sentence structures. But a pattern such as NP - V - NP does not mean that the first NP is the "subject" and the second NP is the "object". In fact, it is not easy to define these notions in the Basic Variety except by their apparent similarity to target or source language utterances. So which argument takes which position? We found that a semantic principle obtains based on the control asymmetry between referents of noun phrases. One can rank each argument of a verb by the greater or lesser degree of control that its referent exerts or intends to exert over the referents of the other argument(s). In the English sentence Clive sliced the salami, Clive ranks higher on the control hierarchy than the salami. The semantic constraint is:

SEM1. The NP referent with the highest control comes first (controller first).

Strength of control ranges from clear agent-patient relations (verbs like kick and push) to weak asymmetries (verbs like kiss and meet), to complete absence (as in copular constructions).

Some verbs, notably verbs of saying and giving, take three arguments (four arguments are never observed in the Basic Variety). These verbs are regularly of the "telic" type; that is, their lexical meaning involves two distinct states (see Klein 1994: 79-97). It is crucial that the control relation between the various arguments is not the same in both states. In an utterance like George gave Eva a book there is a first state (the "source" state) in which George is "in control of" the book and is active in bringing about a distinct state (the target state). In the target state Eva rather than George is "in control of" the book. The control status of the NP that refers to the gift is low in both states. The principle "controller first" thus requires that this argument not come first. It does not prescribe, however, whether the controller of the source state or the controller of the target state comes first. "Controller first" must therefore be supplemented by an additional constraint defining the relative weight of source and target state in determining word order:

SEM2. Controller of source state outweighs controller of target state. 
This principle also applies analogously to verbs of saying if we assume that the control of information changes in both states. There is one referent who controls the information in both states, and another referent who controls the information in the target state, but not in the source state. Thus, the speaker comes first, the hearer comes second, and what is said comes last.

The two control constraints are not always operative, either because there is no asymmetry between the NP referents, or because the verb has only one argument. In these cases, the NP's position depends on how information is distributed across an utterance in context - that is, on pragmatic factors. The Basic Variety has two types of pragmatic constraints. They relate to information status - which information in the utterance is new and which is retained from the preceding utterance $(\mathrm{s})$ - or to the topic-focus structure. These two factors must be kept distinct, although in practice they often appear together. The topic-focus structure reflects the fact that part of the utterance defines a set of alternatives (the topic) and selects the appropriate one (the focus). For example, the utterance Eva ate an apple can answer at least three different questions: (1) Who ate an apple? (2) What did Eva eat? (3) What did Eva do?

In (1), the alternatives are the persons who could have eaten an apple (the topic) and the person specified by the NP Eva (the focus). In (2), the topic is the set of things that Eva could have eaten, and apple specifies one of them (the focus). In (3), the set of alternatives comprises all the events involving Eva that could have occurred on that occasion, and the verb phrase specifies the one selected from this set (the focus). (See Klein and von Stutterheim 1987 for more complex cases in which both factors - information introduction and retention as well as topic-focus structure - are combined in individual utterances and entire texts that constitute an answer to a quaestio: an explicit or implicit question.)

Fully fledged languages can mark an expression as a focus or topic expression by specific devices that include intonation, clefting, and special particles. The Basic Variety mainly uses word order:

PRAG. The focus expression comes last (focus last).

If there is only one argument, then this argument has a semantic role. But there is no semantic role called "asymmetry", and so the controller constraints cannot apply. Hence, only PRAG and phrasal constraints interact. If the referent of the NP is topical, then pattern PH1 is used; if it is in the 


\section{Wolfgang Klein}

focus, then pattern PH3 is used. The same constraint stipulates the NP's position in copula constructions. Our model has the advantage of explaining word order without resorting to ill-defined notions like "subject" or "object". It also explains the "topic status" often associated with the notion of "subject".

The other pragmatic factor that influences utterance structure is the "given-new distinction": is what an expression refers to retained from a preceding utterance or is it new? This distinction actually interacts with the topic-focus status. It does not, however, result in a simple word order rule like PRAG, but rather in different types of NPs. These, in turn, are restricted to certain positions indicated by the numbers in the phrasal rules $\mathrm{PH} 1$ to PH3 noted above. Here we find some limited variation within the Basic Variety. In particular, we find some numerals and (rarely) a definiteness marker, usually a demonstrative. We indicate this in the following diagram by optional DET. As a rule, however, nouns are unadorned. This gives us the following main types:

$$
\begin{array}{cc}
\mathrm{NP}_{1} & \mathrm{NP}_{2} \\
\text { proper name } & \text { proper name } \\
\text { (determiner) noun } & \text { (determiner) noun } \\
\text { pronoun } &
\end{array}
$$

Zero (item without phonological features)

The choice among these forms depends on whether a referent is introduced or retained and whether the referring expression is in topic or focus. The most general opposition is between use of a lexical noun (or proper name) and Zero (or a pronoun). The latter is used exclusively to maintain reference in the context of a controller moving from topic to topic in successive utterances. Maintaining semantic role and position (controller first) is thus not in itself sufficient to licence Zero where there are two potential controllers in the previous utterance (and is a further indication that "subject of" is not a Basic Variety function). With names and lexical nouns, position is the sole indicator of the referent's topic/focus status. It follows from the observed distribution that reference maintenance in focus cannot be achieved by pronominal means. So there are clear constraints on how things can be expressed in the Basic Variety, and where, consequently, its speakers might run into problems. These problems are a major source of structural complexities. 


\section{Conflicts}

The Basic Variety is a remarkably elegant and versatile system. Its structure seems to be independent of the learners' source languages (which in the study were Finnish, Punjabi, Italian, Turkish, Moroccan, Arabic, and Spanish) and target languages (which in the study were English, German, Swedish, Dutch, and French). It should thus reflect properties of the learners' underlying language faculty. It actually offers a number of advantages over fully fledged languages. It lacks irregular verbs and other inflectional nuisances. But problems arise when its neat principles come into conflict. The clearest case we noted was in describing a scene from a film. In order to control the learners' message content, we had them watch and describe scenes from Charlie Chaplin's Modern Times. In one of the scenes a girl is accused of stealing a loaf of bread. In the "German" version of the Basic Variety that is, in the version that primarily uses lexical material based on German - this can be easily described by: (4) Mädchen stehle Brot.

There are two nominal arguments. The first is the controller, the second is focussed. These three rules taken together result in an utterance like (4). But the film's plot becomes more convoluted. The speaker now has to express that Charlie (not the girl) stole the bread. The speakers produced: (5a) Charlie stehle Brot. (5b) Brot stehle Charlie. In (5a) the speaker violates the pragmatic constraint PR1 because Charlie is focussed and so should be in final position. In (5b) the speaker violates SEM1 because Charlie is the controller and so should be in first position. The Basic Variety breaks down in such cases. There are two ways to rectify the problem. The first consists of ranking the two principles:

Semantic constraints outweigh pragmatic constraints.

I suspect that native speakers of English have such a ranking principle. They would infallibly consider the first argument to be the controller. Sentence ( $5 b$ ) thus sounds bizarre to a native speakers of English, but much less so to native speakers of German, since in German the controller might easily be in final position. Hence, if there is ambiguity they tend to follow the opposite ranking. Nevertheless, one of the constraints is violated no matter which ranking is chosen. If we adopt the English strategy, it is not clear which argument is in focus. If we adopt the German strategy, it is not clear which element is the controller (though here it is unlikely that the bread is the controller). 


\section{Wolfgang Klein}

The other way to solve the problem is to invent an additional device that allows the speaker to mark either what is in focus or what is the controller. Both the target and the source languages constrain the inventiveness of the Basic Variety. By contrast, the first homines sapientes, though in principle in the same situation, were without a paradigm. They had to invent something new. Natural languages seem to have either used supra-segmental means or to have created a specific segmental expression (a morpheme) to serve as the additional device. This morpheme may be free or attached to one of the relevant words.

Supra-segmental devices are widely used for this purpose. But to the best of my knowledge they only mark an expression as focussed or non-focussed but never as agent, patient, or the like. They serve CI functions, not LC functions.

The other choice, the formation of a specific morpheme, offers both options. It is possible to invent or adopt a morphological focus marker (or a non-focus marker), and it is possible to invent or adopt a morphological controller marker, a patient marker, and so on. In SLA, the first possibility is exemplified by some learners of French who use a particle [se] to mark an element in initial position as focussed. This particle is a precursor of the cleft construction c'est ... que (see Klein and Perdue 1997: 330). The other possibility is tantamount to case marking, either by inflection or by some free morpheme. The controller can thus be marked by a special suffix, the non-controller by a different suffix, non-focus by still another suffix (thus indicating something like "topicness"), and so on. It may be that the relevant marking only occurs when at least two arguments are present (otherwise no confusion arises), but it is also possible that the case role is marked in all occurrences regardless of whether there is a second argument with which it can be confused.

In the case of language acquisition, the learner is not free to choose among these various options and to build his or her own system. Eventually, the learner has to copy what the social environment does regardless of whether he or she really understands it. Adult learners may be somewhat reluctant to do this if they find it difficult and if they don't see the point. This may be one of the reasons why they often get stuck at a certain stage of proficiency. Children normally do not get stuck. This may be because they are better or more willing imitators of things they do not understand. Our ancestors, who first invented inflectional morphology (including case marking), were not influenced by an already existing system. But we have no direct evidence of what they did. All we have are the results of a long pro- 
cess of transformation, elaboration, and reduction. A fully fledged language and its inflectional morphology resemble an old city in which many generations have left their traces. This explains many of the architectural oddities of modern languages. But it does not preclude them from having had quite systematic foundations.

\section{Conclusion}

As Immanuel Kant said two centuries ago, it is the fate of the human mind to be haunted by questions it cannot answer. He did not mention the quest for the origin of language. But it seems like a good candidate. As William Dwight Whitney, the most eminent American linguist of the nineteenth century, put it in 1873 :

No theme in linguistic science is more often and more voluminously treated than this, and by scholars of every grade and tendency; nor any, it may be added, with less profitable result in proportion to the labour expended; the greater part of what is said and written upon it is mere windy talk, the assertion of subjective views which commend themselves to no mind save the one that produces them, and which are apt to be offered with a confidence, and defended with a tenacity, that are in inverse ratio to their acceptableness. this has given the whole question a bad repute among sober-minded philologists (quoted in Jespersen 1921: 412).

This is not encouraging. Still, I do not think, nor did Whitney, that the issue is necessarily beyond all reasonable research. But it is if no clear distinction is made between various notions of language and, as a consequence, various notions of language origin. First, there is the question of how the human language faculty came into existence. It must have been a complicated biological process involving changes in the brain and in some peripheral organs. We do not know when and how all of this occurred or how it came together to create this remarkable faculty. Once available, this faculty had to create the first linguistic system or systems. This is what nineteenth-century linguists usually understood by the origin of language. This also must have been a complex process involving the creation of a lexicon and of various types of compositional rules. It was not until such linguistic systems were available that communication by means of them was possible. This is the third type of language origin and is the sense in which, for example, Herder proposed that language had its origin in poetry. 


\section{Wolfgang Klein}

The coming-into-existence of linguistic communication is probably the most problematic among the three notions of origin, since it involves the interaction of so many different parts of our cognition. The first point I tried to make in this paper was the need to keep these three notions distinct.

The second point relates to the way in which we have access to these origins. All we can study is the present: the more or less elaborate relics of the past and those aspects of past processes that we still can observe. Today we rarely experience the birth of a new cognitive capacity comparable to the human language faculty. This seems to eliminate the second method. But we can experience new forms of communication, such as the introduction of written communication in oral speech communities or the rise of internet communication. These are interesting and important developments with considerable social consequences. But compared with the origin of linguistic communication they are only elaborations of an existing theme. From the linguist's point of view, the second notion of origin is the most interesting. How did and does the human language faculty create linguistic systems? Its typical task today is to replicate existing systems. It enables children and adults to learn languages. But there is evidence that its language-making ability is not lost, both with respect to the creation of lexemes and of rules of composition. And if we want to understand how it worked before, we should try to find out how it works now. 